\title{
Pain Management and CBD Nano Water
}

\author{
Antoine Chevalier* \\ Department of Natural Health, University of Natural Health, USA
}

Submission: October 18, 2017; Published: November 27, 2017

*Corresponding author: Antoine Chevalier, Department of Natural Health, University of Natural Health, USA, Tel: 301-768-7374;

Email: drantoinechevalier@gmail.com

\section{Introduction}

Neuropathic pain is a worldwide epidemic that occurs in 3 to 8 $\%$ of individuals in industrialized countries and is often refractory to existing treatments. The high rate of addiction and overdose has pushed those treating chronic pain to look for alternatives. In July 2017, a 400-page report by the National Academy of Science presented plans to reduce the addiction crisis, which it said was killing 91 people each day.

Drugs currently available to targetneuropathic pain are, at best, moderately effective and include antidepressants, gabapentin, NMDA receptor antagonists, as well as other anticonvulsants, all of which are limited by their sometimes severe, life-threatening adverse-effect profiles. Cannabinoid based drugs are emerging as a promising class of drugs to treat neuropathic pain and have been tested for analgesic effects in a range of chronic pain conditions. Data show that cannabinoids are often effective in individuals with refractory pain receiving concomitant analgesic drugs. Data from large, well-controlled studies show that cannabinoids are moderately effective in reducing chronic pain and that side effects are less than existing treatments, suggesting that cannabinoids could play a useful role in the management of chronic pain. Like other drugs for neuropathic pain, cannabinoids have a dose titration that is limited by the psycho active side effects of THC when included but not so when CBD isolates are used.

The development of cannabinoid drugs to treat neuropathic pain with improved therapeutic ratios will depend upon the development of cannabinoid treatments with reduced psycho activity. This can be greatly enhanced with improved drug delivery systems such as nano-technology and the use of non-THC containing combinations of Cannabinoids. The endo cannabinoid system, which uses endogenous analogues of cannabinoids known as anamides, has been elucidated over the last decade, demonstrating a significant interface with pain homeostasis.

Exogenous cannabinoids have been demonstrated to be effective in a range of experimental neuropathic pain models, and there is mounting evidence for therapeutic use in human neuropathic pain conditions. This chemical profile bodes well for the future potential of non smoked, orally bio available, non psychoactive cannabinoids delivered using nanotechnology drug delivery systems in the management of neuropathic pain.

Recently, products derived from nano particles have become more innovative and mainstream. Products such as CBD Nano water have been developed by "...breaking the covalent bonds between hydrogen and oxygen atoms which removes water memory before it is purified through several stages of filtering. The water is then infused with cannabinoids (CBD, CBG) as well as vitamins", minerals and terpenes ("Nano particle water"). This results in a "highly medicinal supplemental drink particle" (Nano particle water") and has been demonstrated to relieve symptoms fast. The mechanism being thought to be the rapid transit of the blood brain barrier thereby acting on central as well as peripheral pain centers. One must be careful of immediately believing products that say "nano" in them as the exact mechanisms such as liposomal, micellation, and hydrosome delivery all have their strengths and weaknesses.

Although controversial due to substrate sourcing issues and CSA classification confusion, this process is legal, as "CBD derived from industrial hemp lawfully cultivated in a state that has enacted an industrial hemp pilot research program pursuant to section 7606 of the Farm Act (7 U.S. Code $\S 5940$ - Legitimacy of industrial hemp research) is legal in the state in which the hemp is cultivated" [1]. Hemp oils imported from outside the USA have also been defended on multiple occasions as legal according to the 9th Circuit Court. The summary Agency Issues Legislative Rule in Violation of Administrative".

Procedures Act by Harrison M. Pittman of the National Agricultural Law Center is an excellent overview of the Ninth Circuit Court of Appeals decision in HIA v. DEA. Although anecdotal, increasingly powerful and reproducible testimonials attest to the possibility that CBD Nano water without THC is effective in patients of varying ages and disabilities. Richard A. explained that he is 71 years old and started using CBD water as a treatment for general age ailments. After three weeks, he is 
sleeping better and recovering more quickly after his workouts. Pam also felt the effects of age in the way of arthritis in her hands and knees. She also experienced heartburn.

After utilizing CBD Nano water without THC, she felt that the swelling in her hands have gone down, and swelling in her knees due to surgery has almost completely gone down. Lastly, the heartburn she had been having on a frequent basis has no flared up since starting the regimen of CBD water. All the testimonials mentioned above have solved physical issues, but Richard A. also experienced a decline in anxiety symptoms. CBD has the potential to help in the management of mental and physical illness alike, and that is why it could be a powerful tool in Allopathic medicine. It is an appealing alternative to traditional Western medicine with unpredictable side effects (Figures $1 \& 2$ ).

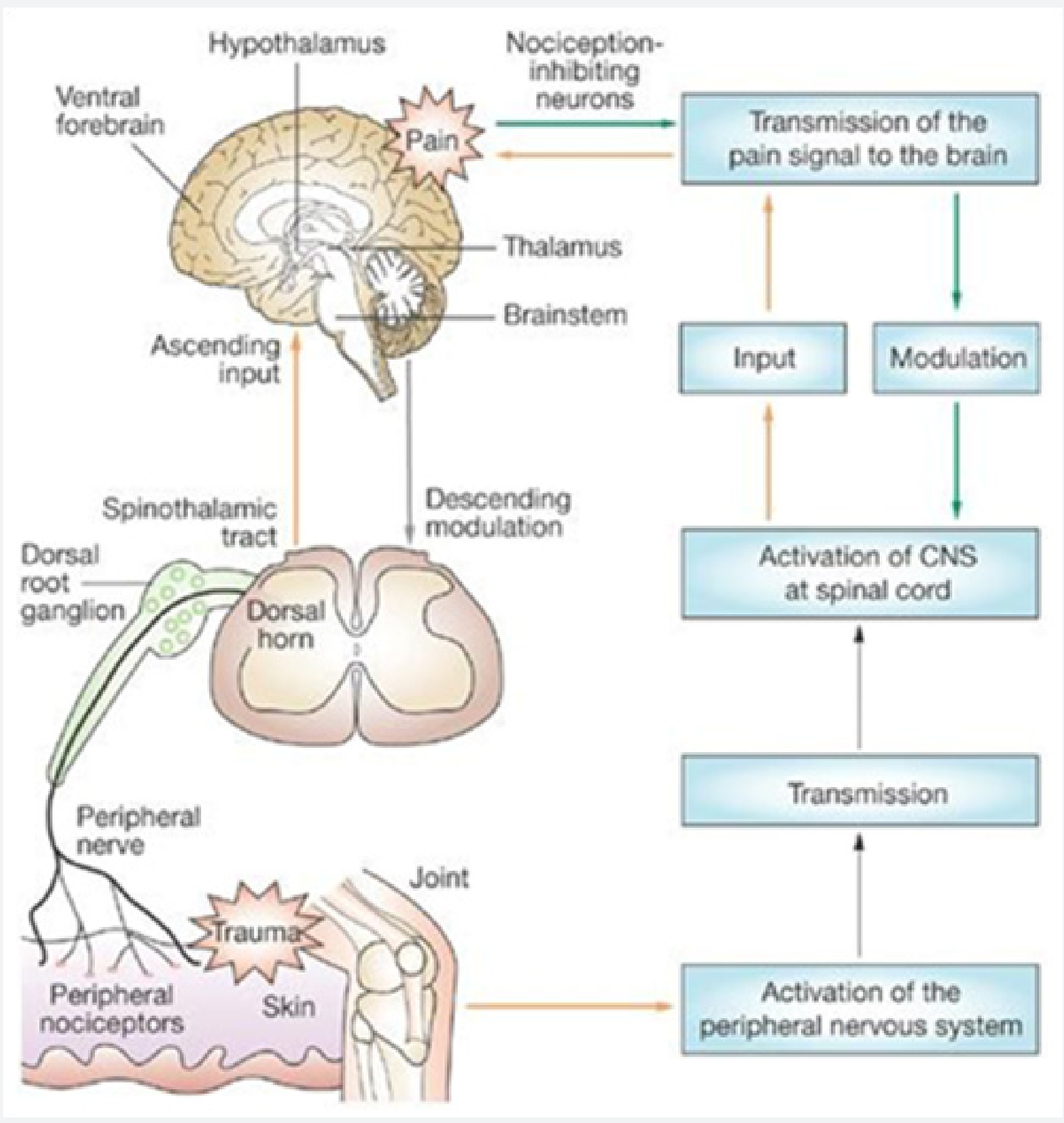

Figure 1: 


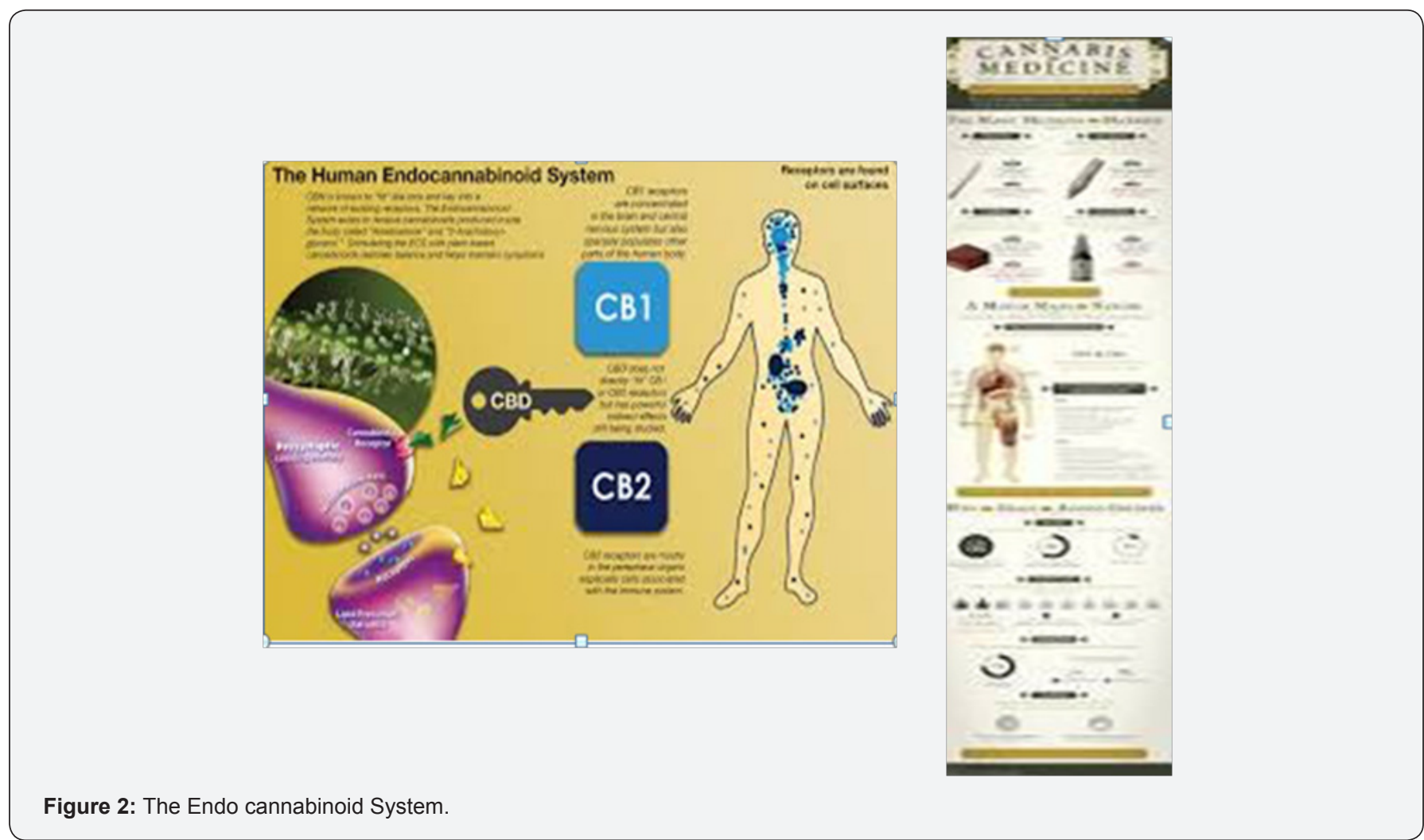

\section{Referencces}

1. Kight R (2017) The legal status of cannabidol, other cannabinoids \& terpenes derived from industrial hemp. Cannabis Law Journal, USA.

2. Weiss L, Zeira, M Mechoulam R Slavin S, Gallily R (2007) Treating or preventing diabetes with cannabidiol. Israel.
3. Ashton JC, Milligan ED (2008) Cannabinoids for the treatment of neuropathic pain: clinical evidence. Curr Opin Investig Drugs 9(1): 6575.

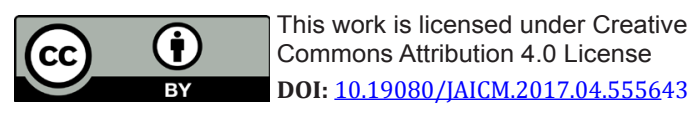

